\title{
A Mitotically Active Uterine Myxoid Leiomyosarcoma Infiltrating The Parametrium And the Ovary
}

\author{
Jawarkar Ashish ${ }^{1}$, Yasmin Momin ${ }^{2}$ \\ ${ }^{1}$ (Assistant Professor, Parul Institute Of Medical Sciences And Research, Vadodara, India) \\ ${ }_{2}^{2}$ (Associate Professor, Grant Medical College And Sir J.J. Group Of Hospitals, Mumbai, India)
}

\begin{abstract}
Myxoid leiomyosarcoma is a rare soft tissue neoplasm of the uterus. Myxoid differentiation coupled with enlarged atypical cells with or without necrosis is termed myxoid leiomyosarcoma. These are mitotically inactive, completely myxoid paucicellular tumors with infiltrative margins. They have a bad prognosis as they are resistant to chemotherapy as well as radiation. Here we present a rare case of uterine myxoid leiomyosarcoma with high mitotic activity infiltrating the parametrium and ovary.
\end{abstract}

Keywords: uterine myxoid leiomyosarcoma, mitoses, resistant, infiltration, paucicellula

\section{Introduction}

Uterine myxoid leiomyosarcoma is a rare neoplasm originally described by King et al in 1982 with less than 20 cases reported in literature. Myxoid differentiation coupled with enlarged atypical cells with or without necrosis and any amount of mitotic activity is termed myxoid leiomyosarcoma. These are mitotically inactive, completely myxoid paucicellular tumors with infiltrative margins. They usually have low mitotic index. Cases with high mitotic rate are extremely rare. We present one such case encountered with infiltration into parametrium and ovary.

\section{Case Report}

63 year old female patient, presented with postmenopausal bleeding, underwent total abdominal hysterectomy for fibroid uterus. We received a total abdominal hysterectomy specimen weighing $1.75 \mathrm{~kg}$. Externally uterus was bosselated. On opening, the endometrial cavity was slit like, distorted by a huge intramural tumor measuring $18 \times 15 \times 8 \mathrm{~cm}$. On serial sectioning, the mass showed areas of hemorrhage, necrosis and a gelatinous appearance with infiltration into parametrium and left ovary (Fig 1). Histological examination showed a myometrial tumor comprised of cells arranged in fascicles and bundles with elongated, blunt ended, hyperchromatic nuclei with prominent nucleoli and moderate amount of eosinophilic cytoplasm. Extensive hypocellular areas composed of myxoid pools with tumor cells floating in it were seen (Fig 2a). Severe atypia, bizarre tumor giant cells and brisk mitoses (80-110/10 high power fields) were noted (Fig 2b). Myometrial, parametrial and ovarian infiltration by tumor was seen.

\section{Discussion}

Myxoid leiomyosarcoma (MLMS) of the uterus is an extreme rare variant of uterine leiomyosarcoma, and only 56 cases were reported from 1982 to 2013 [1]. Uterine MLMS reportedly occurs in wide age range (20-86) years, and common symptoms include atypical genital bleeding, a pelvic mass, and abdominal pain. Grossly MLMS usually has gelatinous appearance and circumscribed border. Microscopically the tumour contains large amount of myxomatous stroma and may invade adjacent tissues. Infiltrating margin is a major factor leading to aggressive behaviour of the tumour [2]. Myxomatous change occurs in 15\% of tumours, more commonly in older women. This entity was first described by King et al. in 1982 with report of six cases. Ten of the 14 previously described cases of myxoid leiomyosarcoma had less than 2 mitoses per $10 \mathrm{HPF}$. However Kunzel et al. described an infiltrative growth pattern, bizarre nuclei and 30 abnormal mitotic figures per 10 HPF. Our case showed nearly 80-110 mitotic figures per 10 high power fields. Traditionally the mitotic count has separated leiomyosarcomas from cellular leiomyomas. For this variant of leiomyosarcoma, the metastatic mitotic count rule does not apply; they tend to recur and metastasize whether mitoses are scanty or numerous. Like other smooth muscle neoplasms, this tumour probably arises from smooth muscle cells of the myometrium or possibly of its blood vessel walls.

\section{Conclusion}

We present this case to emphasize the importance of including myxoid leiomyosarcoma in differential diagnoses of myomas with extensive myxoid change. Also it should be kept in mind that leiomyosarcoma may arise within a leiomyoma. 


\section{References}

[1]. Hiromi Himai et al., Uterine myxoid leiomyosarcoma with tumor emboli extending into the right atrium. Case reports in ob and gy, vol 2015, article id 316262, pages 5

[2]. Burch DM, Tavassoli FA, Myxoid leiomyosarcoma of the uterus. Histopathology 2011 Dec; 59(6):1144-55. doi: 10.1111/j.13652559.2011.04053.x

Fig 1

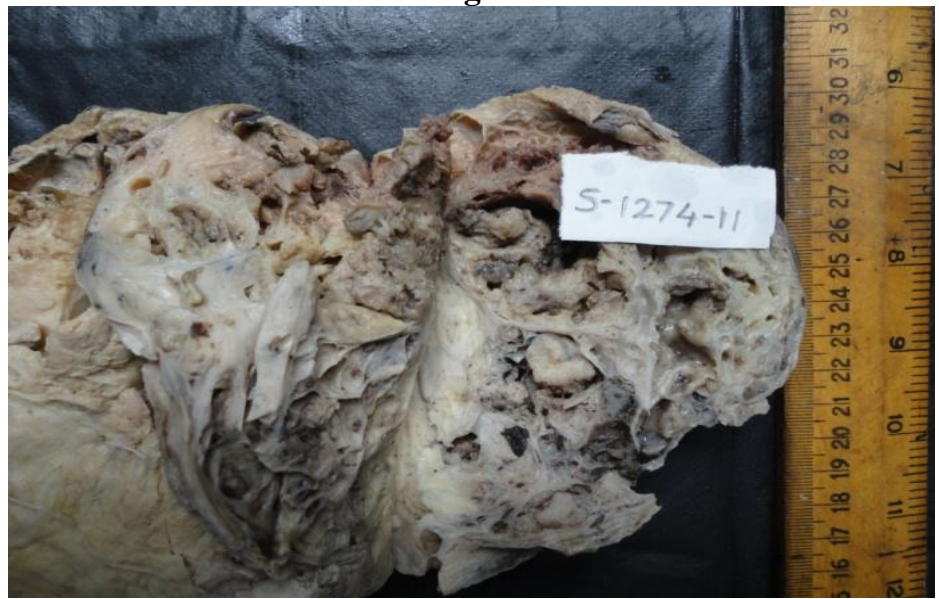

Huge intramural tumor measuring $18 \times 15 \times 8 \mathrm{~cm}$. On serial sectioning, the mass showed areas of hemorrhage, necrosis and a gelatinous appearance with infiltration into parametrium and left ovary

Fig 2a

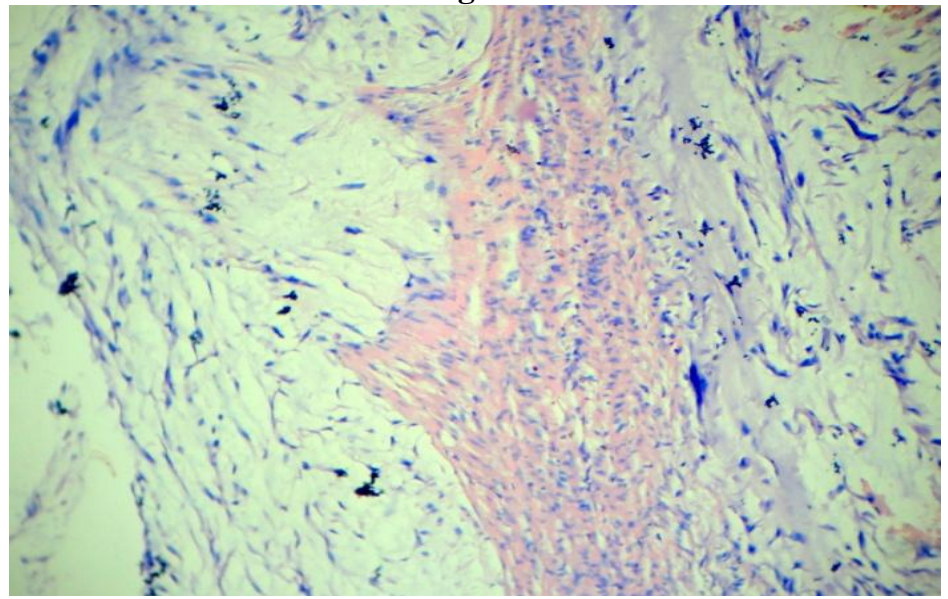

Histological examination showed a myometrial tumor comprised of cells arranged in fascicles and bundles, Extensive hypocellular areas composed of myxoid pools with tumor cells floating in it were seen. Tumor cells had elongated, blunt ended, hyperchromatic nuclei with prominent nucleoli and moderate amount of eosinophilic cytoplasm.

Fig 2b

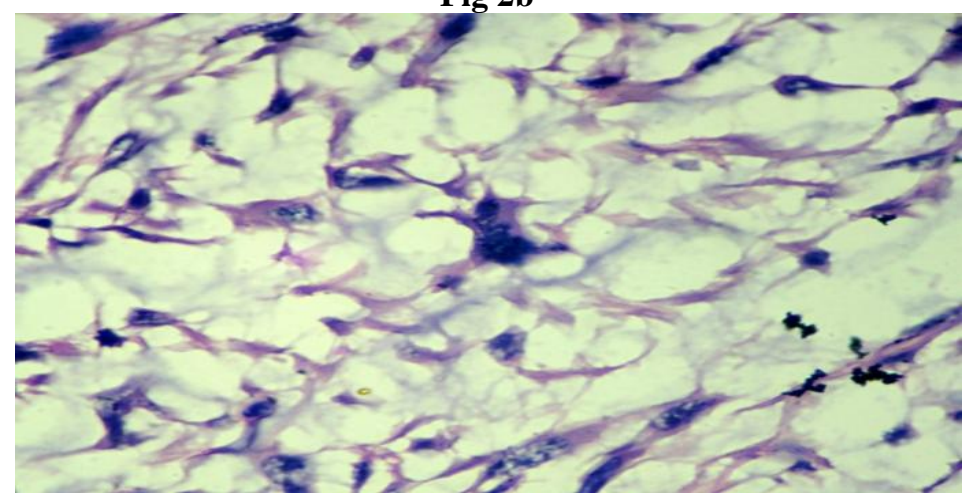

Severe atypia, bizarre tumor giant cells and brisk mitoses (80-110/10 high power fields) were noted 